\title{
A generic global Torelli theorem for certain Horikawa surfaces
}

\author{
Gregory Pearlstein and Zheng Zhang
}

\begin{abstract}
Algebraic surfaces of general type with $q=0, p_{g}=2$ and $K^{2}=1$ were described by Enriques and then studied in more detail by Horikawa. In this paper, we consider a 16-dimensional family of special Horikawa surfaces which are certain bidouble covers of $\mathbb{P}^{2}$. The construction is motivated by that of special Kunev surfaces which are counterexamples for the infinitesimal Torelli and generic global Torelli problems. The main result of the paper is a generic global Torelli theorem for special Horikawa surfaces. To prove the theorem, we relate the periods of special Horikawa surfaces to the periods of certain lattice polarized $K 3$ surfaces using eigenperiod maps and then apply a Torelli type result proved by Laza.
\end{abstract}

\section{Introduction}

There are two particular situations where the period map plays an essential role in the study of moduli spaces, namely principally polarized abelian varieties and (lattice)polarized $K 3$ surfaces. In these cases, the period domains are Hermitian symmetric domains, and the period maps are both injective and dominant. It is an interesting problem to find more examples where the period maps are injective and the images lie in certain Mumford-Tate subdomains which are locally Hermitian symmetric (but the Griffiths infinitesimal period relations may be nontrivial on the ambient period domains). We mention the examples previously studied by Allcock, Carlson and Toledo [ACT02, ACT11], Kondo [Kon00], Borcea [Bor97], Voisin [Voi93], Rohde [Roh09], Garbagnati and Van Geemen [GvG10].

The general problem of determining whether the period map is injective is called a Torelli problem. There are four types of Torelli problems (we follow the terminology of [Cat84a]): local Torelli (whether the differential of the period map is injective), infinitesimal Torelli (local Torelli for the semi-universal deformation), global Torelli (whether the period map is injective) and generic global Torelli (whether the period map is injective over an open dense subset).

Various Torelli theorems have been proved for a large class of varieties (cf. [Cat84a]). However, Kunev [Kyn77] constructed a counterexample for the infinitesimal Torelli and generic global Torelli problems (see also [Cat79, Cat80, Tod80]). Let us briefly recall the construction. Let $C_{1}$ and $C_{2}$ be two smooth plane cubic curves intersecting transversely and $L$ be a general line. Let

Received 28 February 2017, accepted in final form 4 October 2017.

2010 Mathematics Subject Classification 14J10, 14 D07.

Keywords: Torelli problem, Horikawa surfaces, $K 3$ surfaces.

This journal is (C) Foundation Compositio Mathematica 2019. This article is distributed with Open Access under the terms of the Creative Commons Attribution Non-Commercial License, which permits non-commercial reuse, distribution, and reproduction in any medium, provided that the original work is properly cited. For commercial re-use, please contact the Foundation Compositio Mathematica.

The authors acknowledge partial support from NSF Grant DMS-1361120. 


\section{SpeCial Horikawa surfaces}

$X$ be the $(\mathbb{Z} / 2 \mathbb{Z})^{2}$-cover of $\mathbb{P}^{2}$ branched along $C_{1}+C_{2}+L$. Then $X$ is a minimal algebraic surface with $q(X)=0, p_{g}(X)=1$ and $K_{X}^{2}=1$. Following [Cat79], such surfaces $X$ are special Kunev surfaces whose bicanonical maps are Galois covers of $\mathbb{P}^{2}$. The infinitesimal period map and period map for special Kunev surfaces both have 2-dimensional fibers (the rough reason is that the period map only sees the Hodge structures of the intermediate $K 3$ surfaces obtained as the desingularizations of the double planes along $C_{1}+C_{2}$ ).

One may ask whether is it possible to modify the construction or the period map for Kunev surfaces to get a Torelli theorem. Usui [Usu83] and Letizia [Let84] considered the complement of the canonical curve $\Lambda \subset X$ and the mixed Hodge structure $H^{2}(X-\Lambda)$, and proved the infinitesimal mixed Torelli theorem and the generic global mixed Torelli theorem for special Kunev surfaces $X$, respectively. Our idea is to modify the branch data $C_{1}+C_{2}+L$ (see also [Par91a, Definition 2.1]). Specifically, we consider the $(\mathbb{Z} / 2 \mathbb{Z})^{2}$-cover $S$ of $\mathbb{P}^{2}$ along a smooth quintic $C$ together with two generic lines $L_{0}$ and $L_{1}$. The surfaces $S$ are minimal surfaces with $q(S)=0, p_{g}(S)=2$ and $K_{S}^{2}=1$ which have been studied by Horikawa [Hor76]. We shall call such bidouble covers $S$ special Horikawa surfaces. We should remark that these surfaces $S$ are also mentioned in the recent paper [Gar18] by Garbagnati. But her perspective (classify the possible branch loci of a smooth double cover of a $K 3$ surface) is quite different from ours.

Let us explain why we want to modify the branch data in this way. On the one hand, there are two (lattice polarized) $K 3$ surfaces hidden in the construction of a special Horikawa surface $S$ (this is also observed in [Gar18]). Namely, one resolves the singularities of the double cover of $\mathbb{P}^{2}$ branched along $C+L_{1}$ (respectively, $C+L_{0}$ ) and gets $X_{0}$ (respectively, $X_{1}$ ), which is a $K 3$ surface. On the other hand, it is also natural to study the action of the Galois group $(\mathbb{Z} / 2 \mathbb{Z})^{2}$ on the periods of the bidouble covers $S$. We shall show that the eigenperiods (cf. [DK07, $\S 7]$ ) of $S$ are determined by the Hodge structures of the $K 3$ surfaces $X_{0}$ and $X_{1}$ and apply the global Torelli theorem for (lattice polarized) $K 3$ surfaces (a modified version is needed, see the next paragraph) to prove a generic global Torelli theorem for special Horikawa surfaces.

By the work of Catanese [Cat84b] and Pardini [Par91a], the isomorphism classes of the bidouble covers $S$ are determined by the branch data $C+L_{0}+L_{1}$. This can be used to construct the coarse moduli space $\mathcal{M}$ for special Horikawa surfaces (the moduli of special Kunev surfaces has been constructed in a similar manner; see [Usu89]). It also follows that we need the global Torelli theorem [Laz09, Theorem 4.1] for degree 5 pairs $(C, L)$ consisting of plane quintics $C$ and lines $L$ (up to projective equivalence). A key point is that one needs to choose a suitable arithmetic group, as explained in [Laz09, Proposition 4.22].

A typical way to prove a generic global Torelli theorem is to study the infinitesimal variation of Hodge structure and first prove a variational Torelli theorem (cf. [CDT87]). Our approach is different. One advantage is that we are able to describe the open dense subset over which the period map is injective explicitly. We suspect that variational Torelli fails for special Horikawa surfaces (otherwise, by op. cit., the global Torelli theorem holds for any discrete group of the automorphism group of the period domain, as long as the period map is well defined, which seems not true; see also [Hay14]).

After "labeling" the lines $L_{0}$ and $L_{1}$, we obtain a period map (using the period maps for the degree 5 pairs $\left(C, L_{0}\right)$ and $\left.\left(C, L_{1}\right)\right)$ from (a double cover of) the moduli $\mathcal{M}$ of special Horikawa surfaces $S$ to a product of two arithmetic quotients of type IV domains. The period map is generically injective. Therefore, special Horikawa surfaces are along the lines of the examples mentioned at the beginning of the paper. In an ongoing project with Gallardo and Laza, we use this period map as our guide to compactify the moduli space of special Horikawa surfaces. 


\section{G. Pearlstein and Z. Zhang}

Let us say a few words on the structure of the paper. The construction of special Horikawa surfaces is given in Section 2. As is well known (cf. [Hor76]), the canonical model of an algebraic surface with $q=0, p_{g}=2$ and $K^{2}=1$ is a degree 10 hypersurface in the weighted projective space $\mathbb{P}(1,1,2,5)$. We shall give the equations for (the canonical models of) special Horikawa surfaces and use the Griffiths residue to study the decomposition of the Hodge structures. The infinitesimal Torelli problem will be discussed in Section 3. Usui [Usu78] has proved the infinitesimal Torelli theorem for nonsingular weighted complete intersections satisfying certain conditions, which will be checked for special Horikawa surfaces. A second proof will also be included which can be viewed as a boundary case of [Par91b, Theorem 3.1] or [Par98, Theorem 4.2] and might be of independent interest. In Section 4, we discuss the generic global Torelli problem for special Horikawa surfaces and prove the main result.

\section{Special Horikawa surfaces}

Let $C$ be a smooth plane quintic curve. Let $L_{0}$ and $L_{1}$ be two distinct lines which intersect $C$ transversely such that $C \cap L_{0} \cap L_{1}=\emptyset$. We are interested in the bidouble cover $S$ of $\mathbb{P}^{2}$ branched along $C+L_{0}+L_{1}$.

Specifically, the surface $S$ can be constructed in the following way. Take the double cover $\bar{X}_{0}$ of $\mathbb{P}^{2}$ branched along the sextic curve $C+L_{1}$. The surface $\bar{X}_{0}$ is a singular $K 3$ surface with five $A_{1}$ singularities. Let $X_{0}$ be the $K 3$ surface obtained by blowing up the singularities (that is, take the canonical resolution of $\bar{X}_{0}$ ). Denote by $E_{1}, \ldots, E_{5}$ the exceptional curves on $X_{0}$ with self intersection (-2). Set $D_{0}$ to be the preimage of $L_{0}$ in $X_{0}$, and let $D_{1} \subset X_{0}$ be the strict transform of $L_{1}$. Choose $\mathcal{L}:=\mathcal{O}_{X_{0}}\left(D_{1}+E_{1}+\cdots+E_{5}\right)$. By computing the pull-back of $\mathcal{O}_{\mathbb{P}^{2}}(1)$, one sees that $D_{0} \sim 2 D_{1}+E_{1}+\cdots+E_{5}$ and hence $\mathcal{L}^{\otimes 2}=\mathcal{O}_{X_{0}}\left(D_{0}+E_{1}+\cdots+E_{5}\right)$. Now, we take the branched double cover $S_{0}$ of $X_{0}$ along $D_{0}+E_{1}+\cdots+E_{5}$. The exceptional curves $E_{1}, \ldots, E_{5}$ become $(-1)$-curves on $S_{0}$. Contract these $(-1)$-curves, and one obtains a surface $S$. For later use, let us denote by $\sigma_{0}$ the involution of $S$ such that $\bar{X}_{0}=S / \sigma_{0}$. To summarize, we have the following diagram (the left one):
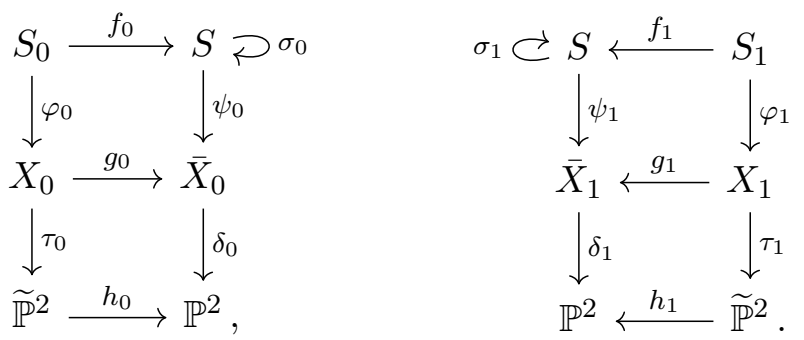

Lemma 2.1. The surface $\psi_{0} \circ \delta_{0}: S \rightarrow \mathbb{P}^{2}$ is a Galois cover with group $(\mathbb{Z} / 2 \mathbb{Z})^{2}$.

Proof. By a result of Zariski, the fundamental group $\pi_{1}\left(\mathbb{P}^{2}-\left(C+L_{0}+L_{1}\right)\right)$ is abelian (see for example [Ful80] or [Cat84b, Theorem 1.6]). Therefore, the covering map $\psi_{0} \circ \delta_{0}$ is defined by a normal subgroup and is Galois. Clearly, the Galois group is an abelian group of order 4. But it cannot be $\mathbb{Z} / 4 \mathbb{Z}$ because otherwise the branch locus $C+L_{0}+L_{1}$ is divisible by 4 in $\operatorname{Pic}\left(\mathbb{P}^{2}\right)$ (or one can directly check that $\sigma_{0}^{2}=\mathrm{id}$ in the group of deck transformations $\operatorname{Deck}\left(S / \mathbb{P}^{2}\right)$ ).

Since the Galois group of the bidouble cover $S$ is $(\mathbb{Z} / 2 \mathbb{Z})^{2}$, there is a symmetric construction for $S$. Namely, one takes the double cover $\delta_{1}: \bar{X}_{1} \rightarrow \mathbb{P}^{2}$ branched along $C+L_{0}$ and resolves the five $A_{1}$ singularities to obtain a $K 3$ surface $g_{1}: X_{1} \rightarrow \bar{X}_{1}$. Call the exceptional curves 


\section{SpeCial Horikawa surfaces}

$F_{1}, \ldots, F_{5} \subset X_{1}$. It can be shown that $\left(\delta_{1} \circ g_{1}\right)^{-1}\left(L_{1}\right)+F_{1}+\cdots+F_{5}$ is divisible by 2 in $\operatorname{Pic}\left(X_{1}\right)$. Let $S_{1}$ be the double cover of $X_{1}$ along $\left(\delta_{1} \circ g_{1}\right)^{-1}\left(L_{1}\right)+F_{1}+\cdots+F_{5}$. The surface $S$ is obtained by contracting the $(-1)$-curves on $S_{1}$. Let us use $\sigma_{1}$ to denote the involution of $S$ with $\bar{X}_{1}=S / \sigma_{1}$. (Note that $\sigma_{0}$ and $\sigma_{1}$ generate the Galois group $(\mathbb{Z} / 2 \mathbb{Z})^{2}$.) See the right part of diagram (2.1).

Proposition 2.2. Let $C$ be a smooth quintic curve, and let $L_{0}$ and $L_{1}$ be two transverse lines with $C \cap L_{0} \cap L_{1}=\emptyset$. Let $S$ be the $(\mathbb{Z} / 2 \mathbb{Z})^{2}$-cover of $\mathbb{P}^{2}$ branched along $C+L_{0}+L_{1}$. Then the surface $S$ is a minimal algebraic surface of general type with $p_{g}(S)=2$ and $K_{S}^{2}=1$. Moreover, $S$ is simply connected, and the canonical bundle $K_{S}$ is ample.

Proof. We use the notation as above. Denote by $\pi: S \rightarrow \mathbb{P}^{2}$ the covering map $\delta_{0} \circ \psi_{0}=\delta_{1} \circ \psi_{1}$. It is clear from the construction that $S$ is smooth. By [Mor88, Lemma 3.2], the canonical bundle $K_{S}$ of the double cover $S$ can be computed as $2 K_{S} \sim \pi^{*} \mathcal{O}_{\mathbb{P}^{2}}(1)$. It follows that $K_{S}$ is ample and $K_{S}^{2}=1$. In particular, $K_{S}$ is big and nef, and hence $S$ is a minimal surface of general type. Now, let us compute $h^{2,0}(S)$, which clearly equals $h^{0}\left(S_{0}, \mathcal{O}_{S_{0}}\left(K_{S_{0}}\right)\right)$. Let $\mathcal{L}=\mathcal{O}_{X_{0}}\left(D_{1}+E_{1}+\cdots+E_{5}\right)$ be the line bundle associated to the double cover $\varphi_{0}: S_{0} \rightarrow X_{0}$ of the $K 3$ surface $X_{0}$. Note that $H^{0}\left(S_{0}, \mathcal{O}_{S_{0}}\left(K_{S_{0}}\right)\right)=H^{0}\left(X_{0}, \varphi_{0 *} \mathcal{O}_{S_{0}}\left(K_{S_{0}}\right)\right)=H^{0}\left(X_{0}, \varphi_{0 *} \varphi_{0}^{*}(\mathcal{L})\right)$. Since $\varphi_{0 *} \varphi_{0}^{*}(\mathcal{L})=\mathcal{L} \otimes$ $\varphi_{0 *}\left(\mathcal{O}_{X_{0}}\right)=\mathcal{L} \otimes\left(\mathcal{O}_{X_{0}} \oplus \mathcal{L}^{-1}\right)=\mathcal{L} \oplus \mathcal{O}_{X_{0}}$, we have $h^{0}\left(S_{0}, \mathcal{O}_{S_{0}}\left(K_{S_{0}}\right)\right)=h^{0}\left(X_{0}, \mathcal{O}_{X_{0}}\right)+h^{0}\left(X_{0}, \mathcal{L}\right)$. Because $D_{1}+E_{1}+\cdots+E_{5}$ is effective and $\left(D_{1}+E_{1}+\cdots+E_{5}\right)^{2}=-2$, the space $H^{0}\left(X_{0}, \mathcal{L}\right)$ is 1-dimensional. Thus $h^{2,0}(S)=h^{0}\left(S_{0}, K_{S_{0}}\right)=2$ (this is also mentioned in [Cat79, Remark 8]). By [Bom73, Theorems 11 and 14] or [Cat84b, Proposition 2.7], the surface $S$ is simply connected.

Algebraic surfaces of general type with $p_{g}=2$ and $K^{2}=1$ have been studied by Horikawa [Hor76]. We call the bidouble covers $S$ constructed above special Horikawa surfaces.

Proposition 2.3. The canonical model of an algebraic surface $Y$ of general type with $q(Y)=0$, $p_{g}(Y)=2$ and $K_{Y}^{2}=1$ is a hypersurface of degree 10 in $\mathbb{P}(1,1,2,5)$. If $K_{Y}$ is ample, then $Y$ is isomorphic to a quasi-smooth hypersurface of degree 10 in $\mathbb{P}(1,1,2,5)$.

Proof. This has been proved in [Hor76, § 2]. See also [KP03, § VII.7].

Remark 2.4. A weighted hypersurface $Y \subset \mathbb{P}$ is quasi-smooth if the associated affine quasicone is smooth outside the vertex 0 (cf. [Dol82]). If, in addition, $\operatorname{codim}_{Y}\left(Y \cap \mathbb{P}_{\text {sing }}\right) \geqslant 2$, then $Y_{\text {sing }}=$ $Y \cap \mathbb{P}_{\text {sing. }}$ (In our case, we have $\mathbb{P}=\mathbb{P}(1,1,2,5)$, which has two singular points $[0,0,1,0]$ and $[0,0,0,1]$, and hence $S_{\text {sing }}=S \cap \mathbb{P}_{\text {sing. }}$.) Moreover, the cohomology $H^{k}(Y, \mathbb{C})$ of a quasi-smooth hypersurface $Y$ admits a pure Hodge structure and can be computed explicitly using a (slightly generalized version of) the Griffiths residue.

We have shown that special Horikawa surfaces $S$ have ample canonical bundles. A natural question is which degree 10 quasi-smooth hypersurfaces in $\mathbb{P}(1,1,2,5)$ do they correspond to.

Proposition 2.5. Let $S$ be a $(\mathbb{Z} / 2 \mathbb{Z})^{2}$-cover of $\mathbb{P}^{2}$ branched over a smooth quintic $C$ and two general lines $L_{0}$ and $L_{1}$ (that is, $S$ is a special Horikawa surface). Then $S$ is isomorphic to a quasi-smooth hypersurface $z^{2}=F\left(x_{0}^{2}, x_{1}^{2}, y\right)$ in $\mathbb{P}(1,1,2,5)=\operatorname{Proj}\left(\mathbb{C}\left[x_{0}, x_{1}, y, z\right]\right)$, where $F$ is a quintic polynomial.

Proof. Denote by $\pi: S \rightarrow \mathbb{P}^{2}$ the covering map $\delta_{0} \circ \psi_{0}=\delta_{1} \circ \psi_{1}$. For $i=0,1$, we let $\Lambda_{i}$ be the reduced inverse image $\pi^{-1}\left(L_{i}\right)$ of $L_{i}$ in $S$. By the proof of Proposition 2.2, we have $\Lambda_{i} \in\left|K_{S}\right|$. Choose a section $x_{i} \in H^{0}\left(S, \mathcal{O}_{S}\left(K_{S}\right)\right)$ which cuts out $\Lambda_{i}$. Clearly, $\left\{x_{0}, x_{1}\right\}$ forms a basis of $H^{0}\left(S, \mathcal{O}_{S}\left(K_{S}\right)\right)$. Since $2 K_{S} \sim \pi^{*} \mathcal{O}_{\mathbb{P}^{2}}(1)$, the covering map $\pi$ is defined by a subspace of $\left|2 K_{S}\right|$. Choose $y \in H^{0}\left(S, \mathcal{O}_{S}\left(2 K_{S}\right)\right)$ such that the subspace of $\left|2 K_{S}\right|$ is generated by $x_{0}^{2}, x_{1}^{2}$ and $y$. Note 


\section{G. Pearlstein and Z. Zhang}

that $y \neq x_{0} x_{1}$. By choosing a suitable $z \in H^{0}\left(S, \mathcal{O}_{S}\left(5 K_{S}\right)\right)$, we assume that the equation for $S$ is $z^{2}=F^{\prime}\left(x_{0}, x_{1}, y\right)$, where $F^{\prime}$ is a weighted homogeneous polynomial of degree 10 in $\mathbb{P}(1,1,2,5)$. (The defining equation must contain $z^{2}$; otherwise, $S$ is not quasi-smooth. In the latter case, we complete the square for $z$, which does not affect the other coordinates.) The ramification locus $\pi$ consists of three components $\left(x_{0}=0\right),\left(x_{1}=0\right)$ and $(z=0)$, which are mapped to $L_{0}, L_{1}$ and $C$, respectively. The proposition then follows.

Alternatively, one considers the action of $\sigma_{i}$ (see diagram (2.1)) on the canonical ring

$$
\bigoplus_{m \geqslant 0} H^{0}\left(S, \mathcal{O}_{S}\left(m K_{S}\right)\right)
$$

and hence on $\mathbb{P}(1,1,2,5)$. Note that the involution $\sigma_{0}$ fixes $\Lambda_{0}$ pointwise and $\sigma_{0}\left(\Lambda_{1}\right)=\Lambda_{1}$, and similarly for the involution $\sigma_{1}$. As in the proof of [Cat79, Theorem 3], one can choose $y$ and $z$ such that $\sigma_{0}$ acts on $\mathbb{P}(1,1,2,5)$ by $\left[x_{0}, x_{1}, y, z\right] \mapsto\left[-x_{0}, x_{1}, y, z\right]$. Since $\sigma_{0}$ acts on $S$, we assume that the defining equation for $S$ is an eigenvector for $\sigma_{0}$. It is not difficult to see that only even powers of $x_{0}$ can appear in the defining equation. Next, one normalizes the equation and gets $z^{2}=F^{\prime}\left(x_{0}, x_{1}, y\right)$. Since $F^{\prime}$ is a weighted homogeneous polynomial of degree 10 , the variable $x_{1}$ also occurs only in even powers.

Remark 2.6. (i) Because $S$ is quasi-smooth, the quintic $F$ must contain $y^{5}$ (more generally, see [FPR17, Theorem 3.3]).

(ii) We have shown that a special Horikawa surface $S$ is isomorphic to a quasi-smooth hypersurface in $\mathbb{P}(1,1,2,5)$ with the equation $z^{2}=F\left(x_{0}^{2}, x_{1}^{2}, y\right)$. The covering map $S \rightarrow \mathbb{P}^{2}$ is given by $\left[x_{0}, x_{1}, y, z\right] \mapsto\left[x_{0}^{2}, x_{1}^{2}, y\right]$. The Galois group is generated by $\sigma_{x_{0}}:\left[x_{0}, x_{1}, y, z\right] \mapsto$ $\left[-x_{0}, x_{1}, y, z\right]$ and $\sigma_{x_{1}}:\left[x_{0}, x_{1}, y, z\right] \mapsto\left[x_{0},-x_{1}, y, z\right]$, which correspond to $\sigma_{0}$ and $\sigma_{1}$, respectively.

(iii) Special Horikawa surfaces have moduli dimension $\left(\left(\begin{array}{c}2+5 \\ 2\end{array}\right)-1\right)+2+2-8=16$ (dimension of moduli for plane quintics together with two lines minus dimension of $\mathrm{PGL}(3))$. The dimension of the moduli for degree 10 quasi-smooth hypersurfaces in $\mathbb{P}(1,1,2,5)=\operatorname{Proj}\left(\mathbb{C}\left[x_{0}, x_{1}, y, z\right]\right)$ cut out by $z^{2}=F\left(x_{0}^{2}, x_{1}^{2}, y\right)$ is $\left(\begin{array}{c}2+5 \\ 2\end{array}\right)-5=16$ (dimension of the quintic polynomials $F$ minus dimension of the subgroup of the automorphism group of $\mathbb{P}(1,1,2,5)$ acting on $z^{2}=F\left(x_{0}^{2}, x_{1}^{2}, y\right)$, a semidirect product of the group consisting of the elements $\left[x_{0}, x_{1}, y, z\right] \mapsto\left[a x_{0}, b x_{1}, c y+d x_{0}^{2}+e x_{1}^{2}, z\right]$ with the group generated by $\left.\left[x_{0}, x_{1}, y, z\right] \mapsto\left[x_{1}, x_{0}, y, z\right]\right)$.

Now, let us study how the Galois group $(\mathbb{Z} / 2 \mathbb{Z})^{2}$ acts on the Hodge structures of special Horikawa surfaces $S$. We view $S$ as a degree 10 quasi-smooth hypersurface in $\mathbb{P}(1,1,2,5)$ cut out by the equation $z^{2}=F\left(x_{0}^{2}, x_{1}^{2}, y\right)$. We choose the Kähler form corresponding to one of the canonical curves $\left(x_{j}=0\right)$ (which is the reduced inverse image of $\left.L_{j}\right)$ and describe the primitive cohomology using the Griffiths residue method.

Proposition 2.7. Let $Y$ be a quasi-smooth hypersurface of degree $d$ in a weighted projective space $\mathbb{P}\left(a_{0}, a_{1}, \ldots, a_{n}\right)$. That is, $Y$ is given by a weighted homogeneous polynomial $G\left(z_{0}, z_{1}\right.$, $\left.\ldots, z_{n}\right)$ of degree $d$ whose partial derivatives have no common zero other that the origin. Let

$$
E=\sum_{i=0}^{n} z_{i} \frac{\partial}{\partial z_{i}}
$$

be the Euler vector field. Let $d V=d z_{0} \wedge \cdots \wedge d z_{n}$ be the Euclidean volume form, and let $\Omega=i(E) d V$ (where $i$ denotes interior multiplication) be the projective volume form (which has 


\section{SpeCial Horikawa surfaces}

degree $\left.a_{0}+\cdots+a_{n}\right)$. Consider expressions of the form

$$
\Omega(A)=\frac{A \cdot \Omega}{G^{q}},
$$

where $A$ is a homogeneous polynomial whose degree is such that $\Omega(A)$ is homogeneous of degree 0 . Then the Poincaré residues of $\Omega(A)$ span $F^{n-q} H_{\text {prim }}^{n-1}(Y, \mathbb{C})$, where $F^{\bullet}$ denotes the Hodge filtration. Moreover, the residue lies in $F^{n-q+1}$ if and only if $A$ lies in the Jacobian ideal $J_{G}$ of $G$ (the ideal generated by the first partial derivatives of $G$ ).

Proof. This is [ACT11, Proposition 1.2]. See also [Dol82].

Proposition 2.8. Let $S \in \mathbb{P}(1,1,2,5)$ be a quasi-smooth hypersurface of degree 10 given by $z^{2}=F\left(x_{0}^{2}, x_{1}^{2}, y\right)$. Set $\sigma_{0}$ and $\sigma_{1}$ to be the automorphisms defined by $\left[x_{0}, x_{1}, y, z\right] \mapsto\left[-x_{0}, x_{1}, y, z\right]$ and $\left[x_{0}, x_{1}, y, z\right] \mapsto\left[x_{0},-x_{1}, y, z\right]$, respectively. Let $\chi_{0}$ and $\chi_{1}$ be the corresponding characters of the Galois group defined by $\chi_{0}\left(\sigma_{0}\right)=1$ and $\chi_{0}\left(\sigma_{1}\right)=-1$, and similarly for $\chi_{1}$. Then we have the following decomposition of Hodge structures:

$$
H_{\text {prim }}^{2}(S, \mathbb{Q})=H_{\chi_{0}}^{2}(S, \mathbb{Q}) \oplus H_{\chi_{1}}^{2}(S, \mathbb{Q}),
$$

where $H_{\chi_{l}}^{2}(S, \mathbb{Q})$ is the eigenspace corresponding to $\chi_{l}$ for $l=0,1$. Moreover, $H_{\chi_{l}}^{2}(S, \mathbb{Q})(l=0,1)$ has Hodge numbers $[1,14,1]$.

Proof. We use the notation of Proposition 2.7. The decomposition is obtained by a Griffiths residue calculus. Specifically, let $G=F\left(x_{0}^{2}, x_{1}^{2}, y\right)-z^{2}$. Take a basis for $H_{\text {prim }}^{2}(S, \mathbb{C})$ consisting of the forms $\operatorname{Res}\left((A \cdot \Omega) / G^{q}\right)$ with $A$ being certain monomials $x_{0}^{i} x_{1}^{j} y^{k}$. The cohomology group $H^{2,0}(S)$ (respectively, $H_{\text {prim }}^{1,1}(S), H^{0,2}(S)$ ) corresponds to $q=1$ (respectively, $q=2, q=3$ ), and hence $i+j+2 k=1$ (respectively, $i+j+2 k=11, i+j+2 k=21$ ). In particular, $i+j$ must be odd. It follows that only the characters $\chi_{0}$ and $\chi_{1}$ appear in the decomposition of $H_{\text {prim }}^{2}(S, \mathbb{C})$ (and hence $H_{\text {prim }}^{2}(S, \mathbb{Q})$ ). The eigenspace $H_{\chi_{0}}^{2}(S, \mathbb{Q})$ is a sub-Hodge structure because $H_{\chi_{0}}^{2}(S, \mathbb{Q})=\operatorname{ker}\left(\sigma_{0}^{*}-\mathrm{id}\right)\left(=\operatorname{ker}\left(\sigma_{1}^{*}+\mathrm{id}\right)\right)$, and similarly for $H_{\chi_{1}}^{2}(S, \mathbb{Q})$. The claim on Hodge numbers can be checked for a special surface $z^{2}=x_{0}^{10}+x_{1}^{10}+y^{5}$ (consider the induced action of $\sigma_{0}$ or $\sigma_{1}$ on the polarized variation of rational Hodge structure with fibers $\left.H_{\text {prim }}^{2}(S, \mathbb{Q})\right)$.

Remark 2.9. Let $X_{0}$ and $X_{1}$ be the $K 3$ surfaces associated to a special Horikawa surface $S$ in diagram (2.1). One can show that $T_{\mathbb{Q}}(S) \cong T_{\mathbb{Q}}\left(X_{0}\right) \oplus T_{\mathbb{Q}}\left(X_{1}\right)$, where $T_{\mathbb{Q}}=\operatorname{Tr} \otimes \mathbb{Q}$ denotes the rational transcendental lattice. Specifically, we consider the induced actions $\sigma_{0}^{*}$ and $\sigma_{1}^{*}$ on $T_{\mathbb{Q}}(S)$. The space $T_{\mathbb{Q}}(S)$ decomposes as a direct sum of the eigenspaces of $\sigma_{0}^{*}$ with eigenvalues 1 and -1 . By [Shi86, Proposition 5], we have $T_{\mathbb{Q}}\left(X_{0}\right) \cong T_{\mathbb{Q}}(S)^{\sigma_{0}^{*}}$ and $T_{\mathbb{Q}}\left(X_{1}\right) \cong T_{\mathbb{Q}}(S)^{\sigma_{1}^{*}}$ (where $T_{\mathbb{Q}}(S)^{\sigma_{l}^{*}}$ denotes the invariant part). Proposition 2.8 allows us to identify $T_{\mathbb{Q}}(S)^{\sigma_{1}^{*}}$ with the $(-1)$-eigenspace of $\sigma_{0}^{*}$, which completes the proof. Generically, $T_{\mathbb{Q}}(S)$ has Hodge numbers $[2,28,2]$ (one applies [Moo93, Proposition 4] to $z^{2}=x_{0}^{10}+x_{1}^{10}+y^{5}$ to see that the generic Picard number is 1 ) and $T_{\mathbb{Q}}\left(X_{l}\right)$ has Hodge numbers $[1,14,1]$ (cf. [Laz09, Corollary 4.15]) for $l=0,1$.

\section{The infinitesimal Torelli theorem}

We shall show in this section that (unlike for special Kunev surfaces [Kyn77, Cat79, Tod80]) the infinitesimal Torelli holds for special Horikawa surfaces $S$. 


\section{G. Pearlstein and Z. Zhang}

Theorem 3.1. Let $S$ be a bidouble cover of $\mathbb{P}^{2}$ branched along a smooth quintic $C$ and two transverse lines $L_{0}$ and $L_{1}$ with $C \cap L_{0} \cap L_{1}=\emptyset$. The natural map

$$
p: H^{1}\left(S, \mathcal{T}_{S}\right) \rightarrow \operatorname{Hom}\left(H^{0}\left(S, \omega_{S}\right), H^{1}\left(S, \Omega_{S}^{1}\right)\right),
$$

given by the cup product, is injective.

Proof. Usui [Usu78] has proved the infinitesimal Torelli theorem for the periods of holomorphic $d$-forms on certain $d$-dimensional complete intersections $(d \geqslant 2)$ in certain weighted projective spaces. One can check that the conditions in [Usu78, Theorem 2.1] are satisfied for the special Horikawa surface $S$ and then apply the theorem. Specifically, by Proposition 2.5, the surface $S \subset$ $\mathbb{P}(1,1,2,5)$ is defined by $z^{2}=F\left(x_{0}^{2}, x_{1}^{2}, y\right)$, and it is not difficult to verify that $z^{2}-F\left(x_{0}^{2}, x_{1}^{2}, y\right)$, $x_{0}, x_{1}, y$ forms a regular sequence for $\mathbb{C}\left[x_{0}, x_{1}, y, z\right]$. (One can apply [Usu78, Proposition 3.1] to check the conditions and prove the infinitesimal Torelli theorem for the periods of holomorphic 2 -forms on any smooth hypersurface with ample canonical bundle in $\mathbb{P}(1,1,2,5)$.)

Pardini [Par91b, Theorem 3.1], [Par98, Theorem 4.2] has considered the infinitesimal Torelli problem for certain abelian covers (including bidouble covers). The conditions of her theorems do not hold for special Horikawa surfaces $S$, but the strategy still works. (In particular, we need the notion of the prolongation bundle discussed in $[$ Par98, §2].) Let us sketch the proof, which might be useful for finding more boundary cases of Pardini's theorems.

Proof. The idea is to decompose the infinitesimal Torelli map

$$
p: H^{1}\left(S, \mathcal{T}_{S}\right) \rightarrow \operatorname{Hom}\left(H^{0}\left(S, \omega_{S}\right), H^{1}\left(S, \Omega_{S}^{1}\right)\right)
$$

using the Galois group action. The first step is to figure out the building data (see [Cat84b, $\S 2$ ] and [Par91a, Definition 2.1]) of the bidouble cover $\pi: S \rightarrow \mathbb{P}^{2}$. The (reduced) branch locus of $\pi$ consists of three irreducible components:

$$
D_{0}:=L_{0}, \quad D_{1}:=L_{1} \quad \text { and } \quad D_{z}:=C .
$$

Let $\sigma_{0}$ and $\sigma_{1}$ be the involutions in diagram (2.1). Let $\sigma_{z}:=\sigma_{0} \circ \sigma_{1}$. The Galois group $G$ of the abelian cover $S \rightarrow \mathbb{P}^{2}$ consists of id, $\sigma_{0}, \sigma_{1}, \sigma_{z}$. Let $\chi_{0}, \chi_{1}, \chi_{z}$ be the corresponding nontrivial characters of $G$ (and we shall denote the character group by $G^{*}$ ). Write

$$
\pi_{*} \mathcal{O}_{S}=\mathcal{O}_{\mathbb{P}^{2}} \oplus \mathcal{L}_{\chi_{0}}^{-1} \oplus \mathcal{L}_{\chi_{1}}^{-1} \oplus \mathcal{L}_{\chi_{z}}^{-1},
$$

where $\mathcal{L}_{\chi}^{-1}$ denotes the eigensheaf on which $G$ acts via the character $\chi$. One easily verifies that $\mathcal{L}_{\chi_{0}}=\mathcal{L}_{\chi_{1}}^{\chi}=\mathcal{O}_{\mathbb{P}^{2}}(3)$ and $\mathcal{L}_{\chi z}=\mathcal{O}_{\mathbb{P}^{2}}(1)$.

Next, we compute the direct images of various sheaves. Let $D=D_{0}+D_{1}+D_{z}$. For the trivial character $\chi=1$, define $\Delta_{1}=D$. Set

$$
\Delta_{\chi_{0}}:=D_{0}, \quad \Delta_{\chi_{1}}:=D_{1}, \quad \Delta_{\chi_{z}}:=D_{z} .
$$

We also let

$$
D_{1,1^{-1}}:=\emptyset, \quad D_{\chi_{0}, \chi_{0}^{-1}}:=D_{1}+D_{z}, \quad D_{\chi_{1}, \chi_{1}^{-1}}:=D_{0}+D_{z}, \quad D_{\chi_{z}, \chi_{z}^{-1}}:=D_{0}+D_{1} .
$$

(For every pair of characters $\chi, \phi \in G^{*}$, the divisor $D_{\chi, \phi}$ is defined in [Par91b] and [Par98]. The fundamental relations of the building data are $\mathcal{L}_{\chi}+\mathcal{L}_{\phi} \equiv \mathcal{L}_{\chi \phi}+D_{\chi, \phi}$; see [Par91a, Theorem 2.1].) For any character $\chi$, we have (see [Par91a, Proposition 4.1])

$$
\begin{aligned}
& -\left(\pi_{*} \mathcal{T}_{S}\right)^{(\chi)}=\mathcal{T}_{\mathbb{P}^{2}}\left(-\log \Delta_{\chi}\right) \otimes \mathcal{L}_{\chi}^{-1}\left(\text { in particular, }\left(\pi_{*} \mathcal{T}_{S}\right)^{(\mathrm{inv})}=\mathcal{T}_{\mathbb{P}^{2}}(-\log D)\right) \\
& -\left(\pi_{*} \Omega_{S}^{1}\right)^{(\chi)}=\Omega_{\mathbb{P}^{2}}^{1}\left(\log D_{\chi, \chi^{-1}}\right) \otimes \mathcal{L}_{\chi}^{-1}\left(\text { in particular, }\left(\pi_{*} \Omega_{S}^{1}\right)^{(\mathrm{inv})}=\Omega_{\mathbb{P}^{2}}^{1}\right)
\end{aligned}
$$




\section{SPECIAL HorikaWA SURFACES}

$-\left(\pi_{*} \omega_{S}\right)^{(\chi)}=\omega_{\mathbb{P}^{2}} \otimes \mathcal{L}_{\chi^{-1}}\left(\right.$ in particular, $\left.\left(\pi_{*} \omega_{S}\right)^{(\text {inv })}=\omega_{\mathbb{P}^{2}}\right)$.

Since the map $\pi$ is finite, for every coherent sheaf $\mathcal{F}$ on $S$ one has $H^{k}(S, \mathcal{F})=H^{k}\left(\mathbb{P}^{2}, \pi_{*} \mathcal{F}\right)$ $(k=0,1,2)$. In particular, we have

$$
H^{1}\left(S, \mathcal{T}_{S}\right)=H^{1}\left(\mathbb{P}^{2}, \pi_{*} \mathcal{T}_{S}\right), \quad H^{1}\left(S, \Omega_{S}\right)=H^{1}\left(\mathbb{P}^{2}, \pi_{*} \Omega_{S}\right), \quad H^{0}\left(S, \omega_{S}\right)=H^{0}\left(\mathbb{P}^{2}, \pi_{*} \omega_{S}\right) .
$$

Combining this with the splittings of $\pi_{*} \mathcal{T}_{S}, \pi_{*} \Omega_{S}$ and $\pi_{*} \omega_{S}$, we obtain the following decompositions:

$$
\begin{aligned}
& H^{1}\left(S, \mathcal{T}_{S}\right)=H^{1}\left(\mathbb{P}^{2}, \mathcal{T}_{\mathbb{P}^{2}}(-\log D)\right) \oplus\left(\underset{\chi \in G^{*} \backslash\{1\}}{\oplus} H^{1}\left(\mathbb{P}^{2}, \mathcal{T}_{\mathbb{P}^{2}}\left(-\log \Delta_{\chi}\right) \otimes \mathcal{L}_{\chi}^{-1}\right)\right), \\
& H^{1}\left(S, \Omega_{S}^{1}\right)=H^{1}\left(\mathbb{P}^{2}, \Omega_{\mathbb{P}^{2}}^{1}\right) \oplus\left(\underset{\chi \in G^{*} \backslash\{1\}}{\oplus} H^{1}\left(\mathbb{P}^{2}, \Omega_{\mathbb{P}^{2}}^{1}\left(\log D_{\chi, \chi^{-1}}\right) \otimes \mathcal{L}_{\chi}^{-1}\right)\right), \\
& H^{0}\left(S, \omega_{S}\right)=H^{0}\left(\mathbb{P}^{2}, \omega_{\mathbb{P}^{2}}\right) \oplus\left(\underset{\chi \in G^{*} \backslash\{1\}}{\oplus} H^{0}\left(\mathbb{P}^{2}, \omega_{\mathbb{P}^{2}} \otimes \mathcal{L}_{\chi^{-1}}\right)\right) .
\end{aligned}
$$

Since the cup product (and hence the infinitesimal Torelli map $p$ ) is compatible with the group action, for characters $\chi, \phi \in G^{*}$, we consider

$$
\begin{aligned}
p_{\chi, \phi}: & H^{1}\left(\mathbb{P}^{2}, \mathcal{T}_{\mathbb{P}^{2}}\left(-\log \Delta_{\chi}\right) \otimes \mathcal{L}_{\chi}^{-1}\right) \\
& \rightarrow \operatorname{Hom}\left(H^{0}\left(\mathbb{P}^{2}, \omega_{\mathbb{P}^{2}} \otimes \mathcal{L}_{\phi^{-1}}\right), H^{1}\left(\mathbb{P}^{2}, \Omega_{\mathbb{P}^{2}}^{1}\left(\log D_{\chi \phi,(\chi \phi)^{-1}}\right) \otimes \mathcal{L}_{\chi \phi}^{-1}\right)\right) .
\end{aligned}
$$

Clearly, one has

$$
p=\underset{\chi, \phi \in G^{*}}{\oplus} p_{\chi, \phi}
$$

Lemma 3.2. The infinitesimal Torelli theorem holds for $S$ (that is, the map $p$ is injective) if and only if $\cap_{\phi \in G^{*}} \operatorname{ker} p_{\chi, \phi}=\{0\}$ for any character $\chi$.

Let us take a closer look at the maps $p_{\chi, \phi}$. For every pairs of characters $\chi, \phi \in G^{*}$ and every section $\xi \in H^{0}\left(\mathbb{P}^{2}, \omega_{\mathbb{P}^{2}} \otimes \mathcal{L}_{\phi^{-1}}\right)$, consider the diagram

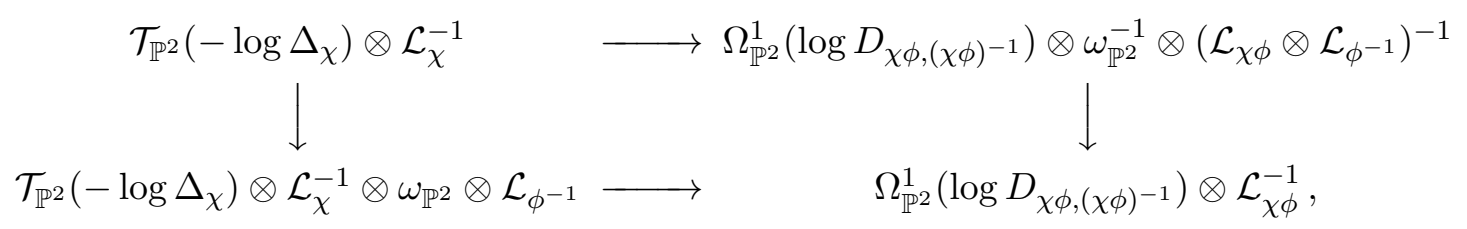

where the vertical maps are given by multiplication by $\xi$ and the horizontal maps are defined by contraction of tensors and by the fundamental relations [Par91a, Theorem 2.1] of the building data (n. b.: there is a canonical isomorphism between $\mathcal{T}_{\mathbb{P}^{2}}\left(-\log D_{\chi \phi,(\chi \phi)^{-1}}\right)$ and $\left.\Omega_{\mathbb{P}^{2}}^{1}\left(\log D_{\chi \phi,(\chi \phi)^{-1}}\right) \otimes\left(\omega_{\mathbb{P}^{2}}\left(D_{\chi \phi,(\chi \phi)^{-1}}\right)\right)^{-1}\right)$. Consider

$$
\begin{aligned}
q_{\chi, \phi}: & H^{1}\left(\mathbb{P}^{2}, \mathcal{T}_{\mathbb{P}^{2}}\left(-\log \Delta_{\chi}\right) \otimes \mathcal{L}_{\chi}^{-1}\right) \\
& \rightarrow H^{1}\left(\mathbb{P}^{2}, \Omega_{\mathbb{P}^{2}}^{1}\left(\log D_{\chi \phi,(\chi \phi)^{-1}}\right) \otimes \omega_{\mathbb{P}^{2}}^{-1} \otimes\left(\mathcal{L}_{\chi \phi} \otimes \mathcal{L}_{\phi^{-1}}\right)^{-1}\right)
\end{aligned}
$$

and

$$
\begin{aligned}
r_{\chi, \phi}: & H^{1}\left(\mathbb{P}^{2}, \Omega_{\mathbb{P}^{2}}^{1}\left(\log D_{\chi, \chi^{-1}}\right) \otimes\left(\omega_{\mathbb{P}^{2}} \otimes \mathcal{L}_{\chi} \otimes \mathcal{L}_{\phi^{-1}}\right)^{-1}\right) \\
& \rightarrow \operatorname{Hom}\left(H^{0}\left(\mathbb{P}^{2}, \omega_{\mathbb{P}^{2}} \otimes \mathcal{L}_{\phi^{-1}}\right), H^{1}\left(\mathbb{P}^{2}, \Omega_{\mathbb{P}^{2}}^{1}\left(\log D_{\chi, \chi^{-1}}\right) \otimes \mathcal{L}_{\chi}^{-1}\right)\right) .
\end{aligned}
$$

Obviously, we have $p_{\chi, \phi}=r_{\chi \phi, \phi} \circ q_{\chi, \phi}$.

Let us analyze the maps $r_{\chi, \phi}$. 


\section{G. Pearlstein and Z. Zhang}

- The maps $r_{1,1}, r_{1, \chi_{0}}, r_{1, \chi_{1}}, r_{1, \chi_{z}}$ are injective; this follows by an explicit computation and Bott's vanishing theorem.

- The maps $r_{\chi_{0}, 1}, r_{\chi_{1}, 1}, r_{\chi_{z}, 1}, r_{\chi_{0}, \chi_{z}}, r_{\chi_{1}, \chi_{z}}$ are zero since $H^{0}\left(\mathbb{P}^{2}, \omega_{\mathbb{P}^{2}} \otimes \mathcal{L}_{\phi^{-1}}\right)=0$.

- The maps $r_{\chi_{0}, \chi_{0}}, r_{\chi_{0}, \chi_{1}}, r_{\chi_{1}, \chi_{0}}, r_{\chi_{1}, \chi_{1}}$ are injective; we use the prolongation bundle of the irreducible components of $D_{\chi, \chi^{-1}}$ defined in $[\mathrm{Par} 98, \S 2]$ and show that if the multiplication map

$$
\begin{aligned}
& H^{0}\left(\mathbb{P}^{2}, \omega_{\mathbb{P}^{2}} \otimes \mathcal{L}_{\phi^{-1}}\right) \otimes\left(\underset{\substack{B \text { irreducible } \\
\text { components of } D_{\chi, \chi^{-1}}}}{\oplus} H^{0}\left(\mathbb{P}^{2}, \mathcal{O}_{\mathbb{P}^{2}}(B) \otimes \omega_{\mathbb{P}^{2}} \otimes \mathcal{L}_{\chi}\right)\right) \\
& \rightarrow \underset{\substack{B \text { irreducible } \\
\text { components of } D_{\chi, \chi^{-1}}}}{\oplus} H^{0}\left(\mathbb{P}^{2}, \mathcal{O}_{\mathbb{P}^{2}}(B) \otimes \omega_{\mathbb{P}^{2}}^{\otimes 2} \otimes \mathcal{L}_{\chi} \otimes \mathcal{L}_{\phi^{-1}}\right)
\end{aligned}
$$

is surjective, then the map $r_{\chi, \phi}$ is injective (cf. [Par98, §3]). As argued in [Par98, Proposition 3.5], the surjectivity of the multiplication map follows from a special case of [EL93, Theorem 2.1].

- The maps $r_{\chi_{z}, \chi_{0}}, r_{\chi_{z}, \chi_{1}}, r_{\chi_{z}, \chi_{z}}$ are injective; we also consider the prolongation bundle and also note that $H^{0}\left(\mathbb{P}^{2}, \mathcal{O}_{\mathbb{P}^{2}}(B) \otimes \omega_{\mathbb{P}^{2}}^{\otimes 2} \otimes \mathcal{L}_{\chi} \otimes \mathcal{L}_{\phi^{-1}}\right)=0$ (with $B$ any irreducible component of $\left.D_{\chi, \chi^{-1}}\right)$ in these cases.

We use the method of [Par91b, §3] to study the maps $q_{\chi, \phi}$ (especially diagram (3.4) and Lemma 3.1). The results are as follows:

- For $\chi=1$, we have ker $q_{1, \chi_{0}} \cap \operatorname{ker} q_{1, \chi_{1}}=\{0\}$ (and hence ker $p_{1, \chi_{0}} \cap \operatorname{ker} p_{1, \chi_{1}}=\{0\}$ ).

- For $\chi=\chi_{0}$, both $q_{\chi_{0}, \chi_{1}}$ and $q_{\chi_{0}, \chi_{z}}$ are injective (and hence $p_{\chi_{0}, \chi_{1}}=r_{\chi_{z}, \chi_{1}} \circ q_{\chi_{0}, \chi_{1}}$ is injective).

- For $\chi=\chi_{1}$, both $q_{\chi_{1}, \chi_{0}}$ and $q_{\chi_{1}, \chi_{z}}$ are injective (and hence $p_{\chi_{1}, \chi_{0}}=r_{\chi_{z}, \chi_{0}} \circ q_{\chi_{1}, \chi_{0}}$ is injective).

- For $\chi=\chi_{z}$, both $q_{\chi_{z}, \chi_{0}}$ and $q_{\chi_{z}, \chi_{1}}$ are injective (and hence $p_{\chi_{z}, \chi_{0}}=r_{\chi_{1}, \chi_{0}} \circ q_{\chi_{z}, \chi_{0}}$ is injective, and $p_{\chi_{z}, \chi_{1}}=r_{\chi_{0}, \chi_{1}} \circ q_{\chi_{z}, \chi_{1}}$ is also injective).

The theorem clearly follows from these observations.

\section{Degree 5 pairs and a generic global Torelli theorem}

Let us review the period map for degree 5 pairs, which will be used later in this section to prove a generic global Torelli theorem for special Horikawa surfaces $S$.

Following [Laz09, Definition 2.1], we call a pair $(C, L)$ consisting of a plane quintic curve $C$ and a line $L \subset \mathbb{P}^{2}$ a degree 5 pair. Two such pairs are equivalent if they are projectively equivalent. We are interested in the degree 5 pairs $(C, L)$ with $C+L$ defining a sextic curve admitting at worst $A D E$ singularities. The coarse moduli space $\mathcal{M}_{A D E}$ is contained in the geometric invariant theory $\left(\right.$ GIT) quotient $\left(\mathbb{P} H^{0}\left(\mathbb{P}^{2}, \mathcal{O}_{\mathbb{P}^{2}}(5)\right) \times \mathbb{P} H^{0}\left(\mathbb{P}^{2}, \mathcal{O}_{\mathbb{P}^{2}}(1)\right)\right) / / \mathrm{SL}_{3}(\mathbb{C})$ (with respect to the linearization $\left.\pi_{1}^{*} \mathcal{O}_{\mathbb{P}^{2}}(1) \otimes \pi_{2}^{*} \mathcal{O}_{\mathbb{P}^{2}}(1)\right)$.

To every degree 5 pair $(C, L)$ such that $C+L$ has at worst $A D E$ singularities, we associate a $K 3$ surface $X_{(C, L)}$ obtained by taking the canonical resolution of the double cover $\bar{X}_{(C, L)}$ of $\mathbb{P}^{2}$ along the sextic $C+L$. The period map for degree 5 pairs $(C, L)$ is defined via the periods of $X_{(C, L)}$. Specifically, one first considers a generic pair $(C, L)$, where $C$ is smooth and $L$ is a transversal. The $K 3$ surface $X_{(C, L)}$ contains five exceptional curves $e_{1}, \ldots, e_{5}$ corresponding to 


\section{SpeCial Horikawa surfaces}

the five intersection points of $C \cap L$ and the strict transform $l^{\prime}$ of $L$. By [Laz09, Proposition 4.12, Definition 4.17], the lattice generated by $\left\{l^{\prime}, e_{1}, \ldots, e_{5}\right\}$ is a primitive sublattice of $\operatorname{Pic}\left(X_{(C, L)}\right)$, and hence $X_{(C, L)}$ is a lattice polarized $K 3$ surface (note that the lattice polarization depends on the labeling of the points of intersection $C \cap L)$.

Notation 4.1. Let $\Lambda$ be an even lattice. We define

$-\Lambda^{*}$ : the dual lattice;

- $A_{\Lambda}=\Lambda^{*} / \Lambda$ : the discriminant group endowed with the induced quadratic form $q_{\Lambda}$;

$-O(\Lambda)$ : the group of isometries of $\Lambda$;

- $O\left(q_{\Lambda}\right)$ : the automorphisms of $A_{\Lambda}$ that preserve the quadratic form $q_{\Lambda}$;

$-O_{-}(\Lambda)$ : the subgroup of isometries of $\Lambda$ of spinor norm 1 (see also [Sca87, § 3.6]);

- $\widetilde{O}(\Lambda)=\operatorname{ker}\left(O(\Lambda) \rightarrow O\left(A_{\Lambda}\right)\right)$ : the group of isometries of $\Lambda$ that induce the identity on $A_{\Lambda}$;

$-O^{*}(\Lambda):=O_{-}(\Lambda) \cap \widetilde{O}(\Lambda)$.

We also introduce

- $\Lambda_{K 3}$ : the $K 3$ lattice $U^{\oplus 3} \oplus E_{8}^{\oplus 2}$ (we denote the bilinear form by $(\cdot, \cdot)_{K 3}$ );

- $M$ : the abstract lattice generated by $\left\{l^{\prime}, e_{1}, \ldots, e_{5}\right\}$ which admits a unique primitive embedding into $\Lambda_{K 3}$ (one can also show that $M$ is the generic Picard group of $K 3$ surfaces $X_{(C, L)}$ and $M \cong U(2) \oplus D_{4}$; see [Laz09, Corollary 4.15, Lemma 4.18]);

- $T=M_{\Lambda_{K 3}}^{\perp}$ : the orthogonal complement of $M$ in $\Lambda_{K 3}$, which is isomorphic to $U \oplus U(2) \oplus$ $D_{4} \oplus E_{8}$

- $\mathcal{D}_{M}:=\left\{\omega \in \mathbb{P}(T \otimes \mathbb{C}) \mid(\omega, \omega)_{K 3}=0,(\omega, \bar{\omega})_{K 3}>0\right\}$, which is the period domain for $M$-polarized $K 3$ surfaces;

- $\mathcal{D}_{M}^{0}$ : a connected component of $\mathcal{D}_{M}$ which is a type IV Hermitian symmetric domain.

Let $\mathcal{U}$ be an open subset of $\mathcal{M}_{A D E}$ parameterizing the generic degree 5 pairs $(C, L)$ with $C$ smooth and with transverse intersections $C \cap L$. Let $\widetilde{\mathcal{U}}$ be the $\mathfrak{S}_{5}$-cover of $\mathcal{U}$ that consists of triples $(C, L, \sigma)$ with $\sigma:\{1, \ldots, 5\} \rightarrow C \cap L$ labelings of $C \cap L$. By [Dol96], there is a period map $\widetilde{\mathcal{U}} \rightarrow \mathcal{D}_{M}^{0} / O^{*}(T)$ sending $(C, L, \sigma)$ to the periods of $X_{(C, L)}$ with the $M$-polarization determined by $\sigma$. By the global Torelli theorem and surjectivity of the period map for $K 3$ surfaces and [Laz09, Proposition 4.14], the period map is birational. Note that there is a natural $\mathfrak{S}_{5}$-action on $\widetilde{\mathcal{U}}$. Moreover, the group $O^{*}(T)$ is a normal subgroup of $O_{-}(T)$ with $O_{-}(T) / O^{*}(T) \cong \mathfrak{S}_{5}$, and the residual $\mathfrak{S}_{5}$-action on $M$ is the permutation of the five points of intersection $C \cap L[\operatorname{Laz} 09$, Proposition 4.22]. (In fact, $O_{-}(T)$ is the monodromy group for the degree 5 pairs.) Thus, the period map is $\mathfrak{S}_{5}$-equivariant and descends to a birational map $\mathcal{U} \rightarrow \mathcal{D}_{M}^{0} / O_{-}(T)$.

The birational map can be extended to a morphism $\mathcal{M}_{A D E} \rightarrow \mathcal{D}_{M}^{0} / O_{-}(T)$ using normalized $M$-polarizations. In particular, one needs to construct an $M$-polarization in the case of nontransversal intersections $C \cap L$. We briefly summarize the construction and refer the readers to [Laz09, §4.2.3] for the details. The construction is a modification of the canonical resolution of singularities of double covers (see [KP03, Theorem III.7.2]). The role of modification is to keep track of the points of intersection $C \cap L$. More precisely, one chooses a labeling of the intersection $\sigma:\{1,2,3,4,5\} \rightarrow C \cap L$ such that for any $p \in C \cap L$, we have $\left|\sigma^{-1}(p)\right|=\operatorname{mult}_{p}(C \cap L)$. Set $Y_{0}=\mathbb{P}^{2}$ and $B_{0}=C+L$. We blow up one singularity at a time (instead of doing simultaneous blow-ups) and do the first five blow-ups in points belonging to $L$. The new branched divisor $B_{i}$ is the strict transform of $B_{i-1}$ together with the exceptional divisor of the blow-up reduced mod 2 . 


\section{G. Pearlstein and Z. Zhang}

The process is repeated until the resulting divisor $B_{N}$ is smooth. Denote the blow-up sequence by $Y_{N} \rightarrow \cdots \rightarrow Y_{i} \rightarrow Y_{i-1} \rightarrow \cdots \rightarrow Y_{0}=\mathbb{P}^{2}$. The double cover $X_{(C, L)}$ of $Y_{N}$ along $B_{N}$ is a minimal resolution of $\bar{X}_{(C, L)}$. Let $p_{i} \in Y_{i-1}(1 \leqslant i \leqslant 5)$ be the centers of the blow-ups which lie on the corresponding strict transforms of $L$. Construct a primitive embedding of $M$ into $\operatorname{Pic}\left(X_{(C, L)}\right)$ by sending $l$ to the class of the reduced preimage of $L$ and sending $e_{i}(1 \leqslant i \leqslant 5)$ to the fundamental cycle associated to the simple singularity of $B_{i-1}$ in the point $p_{i}$. The embedding is normalized in the sense of [Laz09, Definition 4.24], and the construction fits well in families.

By the global Torelli theorem for $K 3$, the surface $X_{(C, L)}$ is unique up to isomorphism. Moreover, one can recover the degree 5 pair $(C, L)$ because the classes $2 l^{\prime}+e_{1}+\cdots+e_{5}$ (which corresponds to the pull-back of $\mathcal{O}_{\mathbb{P}^{2}}(1)$ and determines the covering map and the branched curve) and $l^{\prime}$ (which determines the line $L$ and hence the residue quintic $C$ ) are fixed by the monodromy group $O_{-}(T)$. It follows that the period map

$$
\mathcal{M}_{A D E} \hookrightarrow \mathcal{D}_{M}^{0} / O_{-}(T)
$$

for degree 5 pairs $(C, L)$ with $C+L$ admitting at worst $A D E$ singularities is injective. This is the part we shall need later. For completeness, let us mention that one can verify that the period map is surjective (see [Laz09, § 4.3.1], especially Proposition 4.31). By Zariski's main theorem, the bijective birational morphism between two normal varieties $\mathcal{M}_{A D E} \rightarrow \mathcal{D}_{M}^{0} / O_{-}(T)$ is an isomorphism [Laz09, Theorem 4.1].

Let us focus on the generic global Torelli problem for special Horikawa surfaces. By [Cat84b] or [Par91a, Theorem 2.1], we construct the coarse moduli space $\mathcal{M}$ for special Horikawa surfaces as the open subset of the quotient ${ }^{1}$

$$
\left(\mathbb{P} H^{0}\left(\mathbb{P}^{2}, \mathcal{O}_{\mathbb{P}^{2}}(5)\right) \times \operatorname{Sym}^{2}\left(\mathbb{P} H^{0}\left(\mathbb{P}^{2}, \mathcal{O}_{\mathbb{P}^{2}}(1)\right)\right)\right) / / \mathrm{SL}_{3}(\mathbb{C})
$$

corresponding to triples $\left(C, L, L^{\prime}\right)$ which consist of smooth quintics $C$ and transversals $L$ and $L^{\prime}$ with $C \cap L \cap L^{\prime}=\emptyset$. It is more convenient to work with a double cover $\mathcal{M}^{\prime}$ of $\mathcal{M}$. Specifically, $\mathcal{M}^{\prime}$ is the open subset of the GIT quotient ${ }^{2}$

$$
\left(\mathbb{P} H^{0}\left(\mathbb{P}^{2}, \mathcal{O}_{\mathbb{P}^{2}}(5)\right) \times \mathbb{P} H^{0}\left(\mathbb{P}^{2}, \mathcal{O}_{\mathbb{P}^{2}}(1)\right) \times \mathbb{P} H^{0}\left(\mathbb{P}^{2}, \mathcal{O}_{\mathbb{P}^{2}}(1)\right)\right) / / \mathrm{SL}_{3}(\mathbb{C}),
$$

which parameterizes (up to projective equivalence) triples $\left(C, L_{0}, L_{1}\right)$ with $C$ smooth quintics and $L_{0}$ and $L_{1}$ "labeled" lines which intersect $C$ transversely and satisfy $C \cap L_{0} \cap L_{1}=\emptyset$.

Choose a sufficiently general reference point $b \in \mathcal{M}^{\prime}$ (in particular, we label the two lines), and let $S_{b}$ be the corresponding bidouble cover. Let $V=H_{\text {prim }}^{2}\left(S_{b}, \mathbb{R}\right)$ (with respect to the class of a canonical curve or equivalently a hyperplane section in $\mathbb{P}(1,1,2,5))$. Let $Q$ be the polarization on $V$ defined using cup product. We also write $V_{\mathbb{Q}}=H_{\text {prim }}^{2}\left(S_{b}, \mathbb{Q}\right)$. Consider the action of the Galois group $(\mathbb{Z} / 2 \mathbb{Z})^{2}$ on $S_{b}$, and define $\rho:(\mathbb{Z} / 2 \mathbb{Z})^{2} \rightarrow \operatorname{Aut}(V, Q)$ to be the corresponding representation. We use the notation of diagram (2.1). The Galois group $(\mathbb{Z} / 2 \mathbb{Z})^{2}$ is generated by $\sigma_{0}$ and $\sigma_{1}$. Denote the corresponding characters by $\chi_{0}$ and $\chi_{1}$.

Notation 4.2. We shall use the following notation:

- $\mathcal{D}=\mathcal{D}(V, Q)$ : the period domain parameterizing $Q$-polarized Hodge structures of weight 2 on $V$ with Hodge numbers $[2,28,2]$;

$$
\text { - } \mathcal{D}^{\rho}=\left\{x \in \mathcal{D} \mid \rho(a)(x)=x, \forall a \in(\mathbb{Z} / 2 \mathbb{Z})^{2}\right\}
$$

\footnotetext{
${ }^{1}$ This is the quotient for the linearization induced by $\pi_{1}^{*} \mathcal{O}_{\mathbb{P}^{2}}(1) \otimes \pi_{2}^{*} \mathcal{O}_{\mathbb{P}^{2}}(1) \otimes \pi_{3}^{*} \mathcal{O}_{\mathbb{P}^{2}}(1)$.

${ }^{2}$ This is the quotient with respect to the linearization $\pi_{1}^{*} \mathcal{O}_{\mathbb{P}^{2}}(1) \otimes \pi_{2}^{*} \mathcal{O}_{\mathbb{P}^{2}}(1) \otimes \pi_{3}^{*} \mathcal{O}_{\mathbb{P}^{2}}(1)$.
} 


\section{SpeCial Horikawa surfaces}

- $V(\chi)$ : the eigenspace of $V$ corresponding to the character $\chi$ (it is not difficult to see that the eigenspaces $V(\chi)$ and $V\left(\chi^{\prime}\right)$ are orthogonal with respect to $Q$ if $\left.\chi \neq \chi^{\prime}\right)$;

$-V(\chi)_{\mathbb{Q}}:=V(\chi) \cap V_{\mathbb{Q}}$

- $\mathcal{D}(\chi)$ : the period domain $\mathcal{D}\left(V(\chi),\left.Q\right|_{V(\chi)}\right)$ of type $[1,14,1]$.

Lemma 4.3. There is a natural map $\mathcal{D}^{\rho} \rightarrow \mathcal{D}\left(\chi_{0}\right) \times \mathcal{D}\left(\chi_{1}\right)$ which is injective.

Proof. The lemma follows from Proposition 2.8 and [DK07, $\S 7$. Specifically, only the characters $\chi_{0}$ and $\chi_{1}$ appear in the decomposition of the vector space $V$. Let $V \otimes \mathbb{C}=V^{2,0} \oplus V^{1,1} \oplus V^{0,2}$ be a $Q$-polarized Hodge structure on $V$. The map is defined by sending the Hodge structure to the induced $Q$-polarized Hodge structures on $V\left(\chi_{0}\right)$ and $V\left(\chi_{1}\right)$, which is clearly injective.

Now, we show that the period spaces $\mathcal{D}\left(\chi_{0}\right)$ and $\mathcal{D}\left(\chi_{1}\right)$ are both isomorphic to the period space $\mathcal{D}_{M}$ for $M$-polarized $K 3$ surfaces.

Lemma 4.4. There exists an isomorphism $\left(V\left(\chi_{l}\right)_{\mathbb{Q}}, \frac{1}{2} Q\right) \cong\left(T \otimes \mathbb{Q},(\cdot, \cdot)_{K 3} \otimes \mathbb{Q}\right)$ for $l=0$ or 1 .

Proof. We take $\chi_{0}$ as an example. Let $S=S_{b}$ be the bidouble cover corresponding to the reference point $b \in \mathcal{M}^{\prime}$. We use the notation of diagram (2.1). In particular, by abuse of notation, $\sigma_{0}$ also denotes the involution relative to $\varphi_{0}: S_{0} \rightarrow X_{0}$. Label the points of intersection $C \cap L_{1}$ (the isomorphism we shall describe does not depend on the labeling), and there is a primitive embedding of $M$ (and hence $T$ ) into $H^{2}\left(X_{0}, \mathbb{Z}\right)$. Since $b$ is sufficiently general, $M$ is the Picard lattice of the $K 3$ surface $X_{0}$ [Laz09, Corollary 4.15]. Consider the composition of linear maps

$$
T \otimes \mathbb{Q} \hookrightarrow H^{2}\left(X_{0}, \mathbb{Q}\right) \stackrel{\varphi_{0}^{*}}{\rightarrow} H^{2}\left(S_{0}, \mathbb{Q}\right)^{\sigma_{0}^{*}} .
$$

The map $\varphi_{0}^{*}$ is an isomorphism of vector spaces. Let $D_{1} \subset X_{0}$ be the strict transform of $L_{1}$, and set $E_{1}, \ldots, E_{5}$ to be the exceptional curves. Clearly, $T \otimes \mathbb{Q}$ is the orthogonal complement of $\mathbb{Q}\left[D_{1}\right] \oplus \mathbb{Q}\left[E_{1}\right] \oplus \cdots \oplus \mathbb{Q}\left[E_{5}\right]$ in $H^{2}\left(X_{0}, \mathbb{Q}\right)$. Thus, $T \otimes \mathbb{Q}$ is mapped onto $V\left(\chi_{0}\right)_{\mathbb{Q}}=H_{\text {prim }}^{2}(S, \mathbb{Q})^{\sigma_{0}^{*}}$ by $\varphi_{0}^{*}$. The claim on the bilinear forms is clear.

Corollary 4.5. The period domain $\mathcal{D}\left(\chi_{l}\right)$ has two connected components which are both isomorphic to the 14-dimensional type IV Hermitian symmetric domain $\mathrm{SO}(2,14) / \mathrm{S}(\mathrm{O}(2) \times \mathrm{O}(14))$.

To formulate the theorem, we also need to choose a discrete group. Let $\Gamma_{0}$ and $\Gamma_{1}$ be the discrete subgroups of $\operatorname{Aut}\left(V\left(\chi_{0}\right)_{\mathbb{Q}}, Q\right)$ and $\operatorname{Aut}\left(V\left(\chi_{1}\right)_{\mathbb{Q}}, Q\right)$, respectively, corresponding to $O_{-}(T)$ (using Lemma 4.4). Set $\Gamma$ to be the discrete subgroup in $\operatorname{Aut}\left(V_{\mathbb{Q}}, Q\right)$ which projects onto $\Gamma_{0}$ and $\Gamma_{1}$ under the isomorphism $V_{\mathbb{Q}}=V\left(\chi_{0}\right)_{\mathbb{Q}} \oplus V\left(\chi_{1}\right)_{\mathbb{Q}}$. Let

$$
\mathcal{P}: \mathcal{M} \rightarrow \mathcal{D}^{\rho} / \Gamma
$$

be the period map for special Horikawa surfaces (which are canonically polarized). Because the monodromy group (for the very general base point) is contained in the generic MumfordTate group and $\sigma_{0}^{*}$ and $\sigma_{1}^{*}$ are Hodge tensors for every member of the family, the monodromy representation commutes with the representation $\rho$ (see also [GGK12, III.C, Discussion and Example, pp. 67-68] and [DK07, §7]). By [Laz09, Proposition 4.22], the discrete subgroup $\Gamma$ contains the image of the monodromy representation.

To prove the generic global Torelli theorem for $\mathcal{P}: \mathcal{M} \rightarrow \mathcal{D}^{\rho} / \Gamma$, we consider the map

$$
\mathcal{P}_{0} \times \mathcal{P}_{1}: \mathcal{M}^{\prime} \rightarrow \mathcal{D}_{M}^{0} / O_{-}(T) \times \mathcal{D}_{M}^{0} / O_{-}(T),
$$

which is defined using the period maps for the degree 5 pairs $\left(C, L_{0}\right)$ and $\left(C, L_{1}\right)$. 


\section{G. Pearlstein and Z. Zhang}

Proposition 4.6. The map $\mathcal{P}_{0} \times \mathcal{P}_{1}: \mathcal{M}^{\prime} \rightarrow \mathcal{D}_{M}^{0} / O_{-}(T) \times \mathcal{D}_{M}^{0} / O_{-}(T)$ is generically injective. Proof. By [Laz09, $\S 4.2 .3$ or Theorem 4.1], one can recover a degree 5 pair $(C, L)$ (up to projective equivalence) from the periods of the $K 3$ surface $X_{(C, L)}$. As a result, the isomorphism class of a triple $\left(C, L_{0}, L_{1}\right)$ is determined by the periods of the $K 3$ surfaces $X_{\left(C, L_{0}\right)}$ and $X_{\left(C, L_{1}\right)}$ provided that the quintic $C$ has no nontrivial automorphism. More specifically, we assume $\left(\mathcal{P}_{0} \times\right.$ $\left.\mathcal{P}_{1}\right)\left(C, L_{0}, L_{1}\right)=\left(\mathcal{P}_{0} \times \mathcal{P}_{1}\right)\left(C^{\prime}, L_{0}^{\prime}, L_{1}^{\prime}\right)$. Then there exist $f, g \in \mathrm{PGL}_{3}(\mathbb{C})$ such that $f(C)=C^{\prime}$, $f\left(L_{0}\right)=L_{0}^{\prime}, g(C)=C^{\prime}$, and $g\left(L_{1}\right)=L_{1}^{\prime}$. In particular, one has $\left(g^{-1} \circ f\right)(C)=C$. Because $\operatorname{Aut}(C)=\{\operatorname{id}\}$, we get $f=g$. Thus, the map $\mathcal{P}_{0} \times \mathcal{P}_{1}: \mathcal{M}^{\prime} \rightarrow \mathcal{D}_{M}^{0} / O_{-}(T) \times \mathcal{D}_{M}^{0} / O_{-}(T)$ is generically injective.

Theorem 4.7. The period map $\mathcal{P}: \mathcal{M} \rightarrow \mathcal{D}^{\rho} / \Gamma$ is generically injective.

Proof. Let $\mathcal{P}^{\prime}: \mathcal{M}^{\prime} \rightarrow \mathcal{D}^{\rho, 0} / \Gamma \hookrightarrow \mathcal{D}^{0}\left(\chi_{0}\right) / \Gamma_{0} \times \mathcal{D}^{0}\left(\chi_{1}\right) / \Gamma_{1}$ (where the superscript ${ }^{0}$ denotes the choice of a connected component) be the map sending a labeled triple $\left(C, L_{0}, L_{1}\right)$ to the periods of the corresponding special Horikawa surface $S$ (and to the eigenperiods on the underlying eigenspaces $V\left(\chi_{0}\right)$ and $\left.V\left(\chi_{1}\right)\right)$. We would like to compare $\mathcal{P}^{\prime}$ and $\mathcal{P}_{0} \times \mathcal{P}_{1}$. Note that the transcendental lattice $T$ has been identified with the invariant parts of the underlying vector space $V_{\mathbb{Q}}=H_{\text {prim }}^{2}\left(S_{b}, \mathbb{Q}\right)$ for the involutions $\sigma_{l}^{*}(l=0,1)$ via the natural pull-backs (see Lemma 4.4). We claim that under these identifications, $\mathcal{P}^{\prime}$ coincides with $\mathcal{P}_{0} \times \mathcal{P}_{1}$ up to the order of the periods (after relabeling the two lines, one needs to switch the periods of the two $K 3$ surfaces, but the period of the special Horikawa surface and the eigenperiods remain the same). The reason is that the eigenperiods are obtained by pulling back the holomorphic 2-forms of the two $K 3$ surfaces $X_{0}=X_{\left(C, L_{1}\right)}$ and $X_{1}=X_{\left(C, L_{0}\right)}$ (see diagram (2.1) and Proposition 2.8). Specifically, we choose the orderings for the intersection points of $C \cap L_{1}$ and $C \cap L_{0}$ and define respective primitive embeddings of $M$ and $T$ into $H^{2}\left(X_{0}, \mathbb{Z}\right)$ and $H^{2}\left(X_{1}, \mathbb{Z}\right)$ accordingly. Let $\phi_{l}: \Lambda_{K 3} \stackrel{\cong}{\rightrightarrows} H^{2}\left(X_{l}, \mathbb{Z}\right)$ $(l=0,1)$ be the markings compatible with the primitive embeddings of $M$. The map $\mathcal{P}_{0} \times \mathcal{P}_{1}$ is defined by considering (the $O_{-}(T)$-orbits of) the $K 3$ periods $\phi_{0, \mathbb{C}}^{-1}\left(H^{2,0}\left(X_{0}\right)\right)$ and $\phi_{1, \mathbb{C}}^{-1}\left(H^{2,0}\left(X_{1}\right)\right)$.

Now, let us discuss the (eigen)period map $\mathcal{P}^{\prime}$. We denote $\sigma_{0}$ and $\sigma_{1}$ in diagram (2.1) by $\sigma$ and $\sigma^{\prime}$, respectively, to reflect the fact that they do not depend on how one labels the lines. Let $\chi$ and $\chi^{\prime}$ be the corresponding characters. We now construct a natural $\rho$-marking (cf. [DK07, $\left.\left.\S 7\right]\right)$ on $H_{\text {prim }}^{2}(S, \mathbb{Q})$ using the $K 3$ markings $\phi_{0}$ and $\phi_{1}$. On one hand, Lemma 4.4 gives us $V(\chi) \cong \mathbb{Q} \cong T \otimes \mathbb{Q}$ and $V\left(\chi^{\prime}\right)_{\mathbb{Q}} \cong T \otimes \mathbb{Q}$. On the other hand, one has $T \otimes \mathbb{Q} \stackrel{\phi_{0}}{\rightarrow} H^{2}\left(X_{0}, \mathbb{Q}\right) \stackrel{\cong}{\rightarrow} H^{2}\left(S_{0}, \mathbb{Q}\right)^{\sigma^{*}}$, where the second homomorphism is induced by the natural pull-back map. As in the proof of Lemma 4.4, one can show that the image of the composition (which is clearly injective) is $H_{\text {prim }}^{2}(S, \mathbb{Q})^{\sigma^{*}}$. In other words, we get $T \otimes \mathbb{Q} \cong H_{\text {prim }}^{2}(S, \mathbb{Q})^{\sigma^{*}}$. Similarly, the $K 3$ marking $\phi_{1}$ and the natural pull-back map allow us to identify $T \otimes \mathbb{Q}$ and $H_{\text {prim }}^{2}(S, \mathbb{Q})^{\sigma^{* *}}$. Combining these observations, we get the markings $V(\chi)_{\mathbb{Q}} \cong H^{2}\left(S_{0}, \mathbb{Q}\right)^{\sigma^{*}}$ and $V\left(\chi^{\prime}\right)_{\mathbb{Q}} \cong H^{2}\left(S_{0}, \mathbb{Q}\right)^{\sigma^{\prime *}}$. Taking the preimage of the holomorphic 2-form invariant for $\sigma^{*}\left(\right.$ respectively, $\left.\sigma^{*}\right)$ in $V(\chi)_{\mathbb{Q}} \otimes \mathbb{C}$ (respectively, $V\left(\chi^{\prime}\right)_{\mathbb{Q}} \otimes \mathbb{C}$ ), one obtains the eigenperiod map $\mathcal{P}^{\prime}$. Now, our claim is clear. By Proposition 4.6, generically, $\mathcal{P}^{\prime}$ has degree 2 (depending on the labelings of the lines). As a result, the period map $\mathcal{P}: \mathcal{M} \rightarrow \mathcal{D}^{\rho} / \Gamma$ is generically injective.

Remark 4.8. Assume that the quintic curve $C$ admits a nontrivial automorphism $\sigma$ satisfying $\sigma\left(L_{0}\right) \neq L_{0}$ and $\sigma\left(L_{1}\right) \neq L_{1}$. Then the triples $\left(C, L_{0}, L_{1}\right)$ and $\left(C, \sigma\left(L_{0}\right), L_{1}\right)$ are mapped by the period map $\mathcal{P}$ to the same point.

Remark 4.9. Let $\mathcal{W}$ be the subset of $\left|\mathcal{O}_{\mathbb{P}^{2}}(5)\right| \times\left|\mathcal{O}_{\mathbb{P}^{2}}(1)\right| \times\left|\mathcal{O}_{\mathbb{P}^{2}}(1)\right|$ corresponding to triples $\left(C, L_{0}, L_{1}\right)$ with $C+L_{0}$ and $C+L_{1}$ admitting at worst $A D E$ singularities and $C \cap L_{0} \cap L_{1}=\emptyset$. 


\section{SpeCial Horikawa surfaces}

By taking bidouble covers, we obtain a family of surfaces $\mathcal{S} \rightarrow \mathcal{W}$ with only du Val singularities. By applying a simultaneous resolution to the family $\mathcal{S}$, we obtain a family $\widetilde{\mathcal{S}} \rightarrow \mathcal{W}$ (after a finite base change of $\mathcal{W}$ ) of Horikawa surfaces (which are surfaces of general type with $p_{g}=2$ and $\left.K^{2}=1\right)$. Consider the period map $\mathcal{P}_{0} \times \mathcal{P}_{1}: \mathcal{W} \rightarrow \mathcal{D}_{M}^{0} / O_{-}(T) \times \mathcal{D}_{M}^{0} / O_{-}(T)$. The generic global Torelli theorem holds for this family. Namely, if two generic points in $\mathcal{W}$ have the same image in $\mathcal{D}_{M}^{0} / O_{-}(T) \times \mathcal{D}_{M}^{0} / O_{-}(T)$, then the corresponding triples are projectively equivalent.

\section{ACKNOWLEDGEMEnTS}

The work is partly motivated by the recent project by Green, Griffiths, Laza and Robles on studying degenerations of "H-surfaces" (which are of general type with $p_{g}=K^{2}=2$ ) using Hodge theory. We thank Griffiths for his interest in this paper. We also thank the referee for the valuable comments. Finally, we are grateful to P. Gallardo and R. Laza for several useful discussions.

\section{REFERENCES}

ACT02 D. Allcock, J.A. Carlson and D. Toledo, The complex hyperbolic geometry of the moduli space of cubic surfaces, J. Algebraic Geom. 11 (2002), no. 4, 659-724; doi:10.1090/S1056-3911-02-00314-4.

ACT11 , The moduli space of cubic threefolds as a ball quotient, Mem. Amer. Math. Soc. 209 (2011), no. 985; doi:10.1090/s0065-9266-10-00591-0.

Bom73 E. Bombieri, Canonical models of surfaces of general type, Publ. Math. Inst. Hautes Études Sci. (1973), no. 42, 171-219; doi:10.1007/BF02685880.

Bor97 C. Borcea, K3 surfaces with involution and mirror pairs of Calabi-Yau manifolds, Mirror Symmetry, II, AMS/IP Stud. Adv. Math., vol. 1 (Amer. Math. Soc., Providence, RI, 1997), 717-743.

Cat79 F. Catanese, Surfaces with $K^{2}=p_{g}=1$ and their period mapping, Algebraic Geometry (Proc. Summer Meeting, Univ. Copenhagen, Copenhagen, 1978), Lecture Notes in Math., vol. 732 (Springer, Berlin, 1979), 1-29; doi:10.1007/BFb0066634.

Cat80 The moduli and the global period mapping of surfaces with $K^{2}=p^{g}=1$ : a counterexample to the global Torelli problem, Compos. Math. 41 (1980), no. 3, 401-414; http: //www.numdam.org/item?id=CM_1980_-41_3_401_0.

Cat84a Infinitesimal Torelli theorems and counterexamples to Torelli problems, Topics in Transcendental Algebraic Geometry (Princeton, N.J., 1981/1982), Ann. of Math. Stud., vol. 106 (Princeton Univ. Press, Princeton, NJ, 1984), 143-156.

Cat84b - On the moduli spaces of surfaces of general type, J. Differential Geom. 19 (1984), no. 2, 483-515; doi:10.4310/jdg/1214438689.

CDT87 D. Cox, R. Donagi and L. Tu, Variational Torelli implies generic Torelli, Invent. Math. 88 (1987), no. 2, 439-446; doi:10.1007/BF01388918.

DK07 I. V. Dolgachev and S. Kondō, Moduli of K3 surfaces and complex ball quotients in Arithmetic and Geometry around Hypergeometric Functions, Progr. Math., vol. 260 (Birkhäuser, Basel, 2007), 43-100; doi:10.1007/978-3-7643-8284-1_3.

Dol82 I. V. Dolgachev, Weighted projective varieties, Group Actions and Vector Fields (Vancouver, B.C., 1981), Lecture Notes in Math., vol. 956 (Springer, Berlin, 1982), 34-71; doi:10.1007/BFb0101508.

Dol96 _ Mirror symmetry for lattice polarized K3 surfaces, J. Math. Sci. 81 (1996), no. 3, 25992630; doi:10.1007/BF02362332. 


\section{G. Pearlstein and Z. Zhang}

EL93 L. Ein and R. Lazarsfeld, Syzygies and Koszul cohomology of smooth projective varieties of arbitrary dimension, Invent. Math. 111 (1993), no. 1, 51-67; doi:10.1007/BF01231279.

Enr49 F. Enriques, Le Superficie Algebriche (Nicola Zanichelli, Bologna, 1949).

FPR17 M. Franciosi, R. Pardini and S. Rollenske, Gorenstein stable surfaces with $K_{X}^{2}=1$ and $p_{g}>0$, Math. Nachr. 290 (2017), no. 5-6, 794-814; doi:10.1002/mana. 201600090.

Ful80 W. Fulton, On the fundamental group of the complement of a node curve, Ann. of Math. 111 (1980), no. 2, 407-409; doi:10.2307/1971204.

Gar18 A. Garbagnati, Smooth double covers of K3 surfaces, Ann. Scuola Norm. Sup. Pisa Cl. Sci., to appear; doi:10.2422/2036-2145.201701_013.

GGK12 M. Green, P. Griffiths and M. Kerr, Mumford-Tate groups and domains. their geometry and arithmetic, Ann. of Math. Stud., vol. 183 (Princeton Univ. Press, Princeton, NJ), 2012.

GvG10 A. Garbagnati and B. van Geemen, Examples of Calabi-Yau threefolds parametrised by Shimura varieties, Rend. Semin. Mat. Univ. Politec. Torino 68 (2010), no. 3, 271-287; http://www. seminariomatematico.unito.it/rendiconti/68-3/271.pdf.

Hay14 N. Hayashi, Counterexample to variational Torelli problem for algebraic surfaces of general type with geometric genus two, Kobe J. Math. 31 (2014), no. 1-2, 9-20.

Hor76 E. Horikawa, Algebraic surfaces of general type with small $c_{1}^{2}$. II, Invent. Math. 37 (1976), no. 2, 121-155; doi:10.1007/BF01418966.

Kon00 S. Kondō, A complex hyperbolic structure for the moduli space of curves of genus three, J. reine angew. Math. 525 (2000), 219-232; doi:10.1515/crll.2000.069.

KP03 T. Kappeler and J. Pöschel, KdV \&ु KAM, Ergeb. Math. Grenzgeb. (3), vol. 45 (Springer-Verlag, Berlin, 2003); doi:10.1007/978-3-662-08054-2.

Kyn77 V.I. Kynev, An example of a simply connected surface of general type for which the local Torelli theorem does not hold, C. R. Acad. Bulgare Sci. 30 (1977), no. 3, 323-325.

Laz09 R. Laza, Deformations of singularities and variation of GIT quotients, Trans. Amer. Math. Soc. 361 (2009), no. 4, 2109-2161; doi:10.1090/S0002-9947-08-04660-6.

Let84 M. Letizia, Intersections of a plane curve with a moving line and a generic global Torelli-type theorem for Kunev surfaces, Amer. J. Math. 106 (1984), no. 5, 1135-1146; doi:10.2307/2374276.

Moo93 B. J. J. Moonen, The Picard number of certain algebraic surfaces, J. Pure Appl. Algebra 85 (1993), no. 3, 317-330; doi:10.1016/0022-4049(93)90145-J.

Mor88 D. R. Morrison, On the moduli of Todorov surfaces, Algebraic Geometry and Commutative Algebra, Vol. I (Kinokuniya, Tokyo, 1988), 313-355.

Par91a R. Pardini, Abelian covers of algebraic varieties, J. reine angew. Math. 417 (1991), 191-213; doi:10.1515/crll.1991.417.191.

Par91b_ Infinitesimal Torelli and abelian covers of algebraic surfaces, Problems in the Theory of Surfaces and their Classification (Cortona, 1988), Sympos. Math., vol. 32 (Academic Press, London, 1991), 247-257.

Par98_, On the period map for abelian covers of projective varieties, Ann. Scuola Norm. Sup. Pisa Cl. Sci. 26 (1998), no. 4, 719-735; http://www. numdam.org/item?id=ASNSP_1998_4_26_ 4_719_0.

Roh09 J.C. Rohde, Cyclic coverings, Calabi-Yau manifolds and complex multiplication, Lecture Notes in Math., vol. 1975 (Springer-Verlag, Berlin, 2009); doi:10.1007/978-3-642-00639-5.

Sca87 F. Scattone, On the compactification of moduli spaces for algebraic K3 surfaces, Mem. Amer. Math. Soc. 70 (1987), no. 374; doi:10.1090/memo/0374.

Shi86 T. Shioda, An explicit algorithm for computing the Picard number of certain algebraic surfaces, Amer. J. Math. 108 (1986), no. 2, 415-432; doi:10.2307/2374678.

Tod80 A. N. Todorov, Surfaces of general type with $p_{g}=1$ and $(K, K)=1$. I, Ann. Sci. École Norm. Sup. (4) 13 (1980), no. 1, 1-21; doi:10.24033/asens. 1375. 


\section{SPECIAL HorikaWA SURFACES}

Usu78 S. Usui, Local Torelli theorem for some nonsingular weighted complete intersections, Proc. of the Int. Symp. on Algebraic Geometry (Kyoto Univ., Kyoto, 1977) (Kinokuniya Book Store, Tokyo, 1978), 723-734.

Usu83 , Variation of mixed Hodge structures arising from family of logarithmic deformations, Ann. Sci. École Norm. Sup. (4) 16 (1983), no. 1, 91-107; doi:10.24033/asens. 1441.

Usu89 - Type I degeneration of Kunev surfaces, Astérisque (1989), no. 179-180, 185-243.

Voi93 C. Voisin, Miroirs et involutions sur les surfaces K3, Astérisque (1993), no. 218, 273-323.

Gregory Pearlstein gpearl@math.tamu.edu

Department of Mathematics, Texas A\&M University, College Station, TX 77843-3368, USA

Zheng Zhang Zheng.Zhang-2@Colorado.edu

Department of Mathematics, Texas A\&M University, College Station, TX 77843-3368, USA

Current address: Department of Mathematics, University of Colorado Boulder, Campus Box 395,

Boulder, CO 80309-0395, USA 